\title{
Aesthetic rehabilitation of a patient with an anterior maxillectomy defect, using an innovative single-step, single unit, plastic-based hollow obturator
}

\author{
Vishwas Bhatia', Garima Bhatia² \\ ${ }^{1}$ Department of Prosthodontics, King Khalid University, Abha 61412, Saudi Arabia. \\ ${ }^{2}$ Department of Oral Medicine and Radiology, PDM Dental College and Hospital, Bahadurgarh, Haryana 124507, India.
}

Address for correspondence: Dr. Vishwas Bhatia, Department of Prosthodontics, King Khalid University, Abha 61412, Saudi Arabia. E-mail: vishwas211@yahoo.co.in

\begin{abstract}
What could be better than improving the comfort and quality of life of a patient with a life-threatening disease? Maxillectomy, the partial or total removal of the maxilla in patients suffering from benign or malignant neoplasms, creates a challenging defect for the maxillofacial prosthodontist when attempting to provide an effective obturator. Although previous methods have been described for rehabilitation of such patients, our goal should be to devise one stage techniques that will allow the patient an improved quality of life as soon as possible. The present report describes the aesthetic rehabilitation of a maxillectomy patient by use of a hollow obturator. The obturator is fabricated through a processing technique which is a variation of other well-known techniques, consisting of the use of a single-step flasking procedure to fabricate a single-unit hollow obturator using the lost salt technique. As our aim is to aesthetically and functionally rehabilitate the patient as soon as possible, the present method of restoring the maxillectomy defect is cost-effective, time-saving and beneficial for the patient.
\end{abstract}

Key words:

Aesthetics, maxillectomy, palatal obturator, plastic-based, squamous cell carcinoma

\section{INTRODUCTION}

A conventional surgical excision is the most common method for treatment of a maxillary oral squamous cell carcinoma (SCC). The resulting surgical defect often includes part of the hard palate which results in oro-antral communication. ${ }^{[1]}$ Patients undergoing surgery alone without closure or obturation of the surgical defect face numerous problems in phonetics and mastication secondary to the passage of air, food and liquids into the nasal and maxillary sinus. In addition to the functional

\begin{tabular}{|l|l|}
\hline \multicolumn{2}{|c|}{ Access this article online } \\
\hline Quick Response Code: & Website: \\
\hline & www.parjournal.net \\
\cline { 2 - 2 } & \\
\hline & \\
\hline
\end{tabular}

deficit, there is a marked effect on aesthetics without the presence of an obturator.

An obturator is that component of a prosthesis, which fits into and closes a defect within the oral cavity or another body defect. ${ }^{[2]}$ In the past, various methods have been used to restore the maxillary defect using silicon bulb obturators, implant-supported obturators, and cast metal obturators. This clinical report describes a method for aesthetic rehabilitation of a patient with a partial maxillectomy defect, using a light-weight, single-unit, closed hollow obturator fabricated by an innovative single-step flasking technique using the lost salt method. The technique assists in fabrication of an obturator, which restores aesthetics, function, speech, and dental appearance.

\section{CASE REPORT}

A 47-year-old man, diagnosed with SCC of the palate extending into the maxillary sinus, underwent a partial 
maxillectomy and was subsequently referred to the Department of Prosthodontics and Implantology, Eklavya Dental College and Hospital, Rajasthan, India. Immediate surgical reconstruction was not recommended given the need for further treatment with radiation therapy. External beam postoperative radiotherapy was administered over a period of 6 weeks. The patient tolerated the radiation well and was subsequently referred to possible prosthetic restoration of the oral defect after radiation therapy. Examination revealed a partial maxillectomy defect on the left side crossing the midline. The left side naso-maxillary region was depressed due to bone loss, and this was also evident in extra oral examination. The defect was a Class IV according to the Aramany Classification of Defects $^{[3]}$ [Figure 1]. The patient agreed to have his pictures published and signed the consent form.

Aesthetic rehabilitation can be accomplished either surgically or prosthetically. ${ }^{[4]}$ The choice of rehabilitation depends on the site, size, severity, patient age, and patient preference. Contraindications to surgical reconstruction include advanced age, poor general medical condition, a history of radiation therapy, a complex anatomical defect and the patient's refusal to undergo further surgery. ${ }^{[5]}$

Various modalities for prosthetic reconstruction were discussed with the patient, and he requested an economical solution. The treatment plan therefore was to provide a plastic-based, light-weight obturator to meet the aesthetic demands by replacing bone and teeth while assisting phonetics and mastication.

\section{Procedure}

An irreversible hydrocolloid was used to make an impression of the maxillary defect area after blocking all undercuts with wet gauge. The impression was poured, and the final cast was obtained, on which a custom tray was made using a self-curing autopolymerising resin.

Border molding for recording the soft tissue borders of the defect was carried out using a low-fusing impression compound. Additional silicone was used to make a wash impression, and the final master cast was poured. All undercuts on the cast were blocked out with plaster and wax [Figure 2]. The final denture base and occlusal wax rims were prepared to record maxillomandibular relations. After the maxillomandibular jaw relations had been obtained, the record was articulated, and teeth arrangement was performed. On completion, the wax prosthesis was verified at the trial insertion appointment. The wax prosthesis was invested, and the wax was eliminated [Figure 3]. A sheet of plastic based heat cure acrylic polymer in the dough stage was placed over the defect and the palatal area on the master cast. Pressure then applied to the base of the defect resulted in a cup-shaped depression of acrylic polymer over the defect [Figure 4]. Salt was then used to fill the depression [Figure 5]. Another thin sheet of acrylic polymer was placed, and packing was performed with conventional prosthodontic protocols. Finally, three to four holes were drilled on the palatal surface of the prosthesis covering the bulb [Figure 6]. Warm water was injected through the holes to dissolve and eliminate the

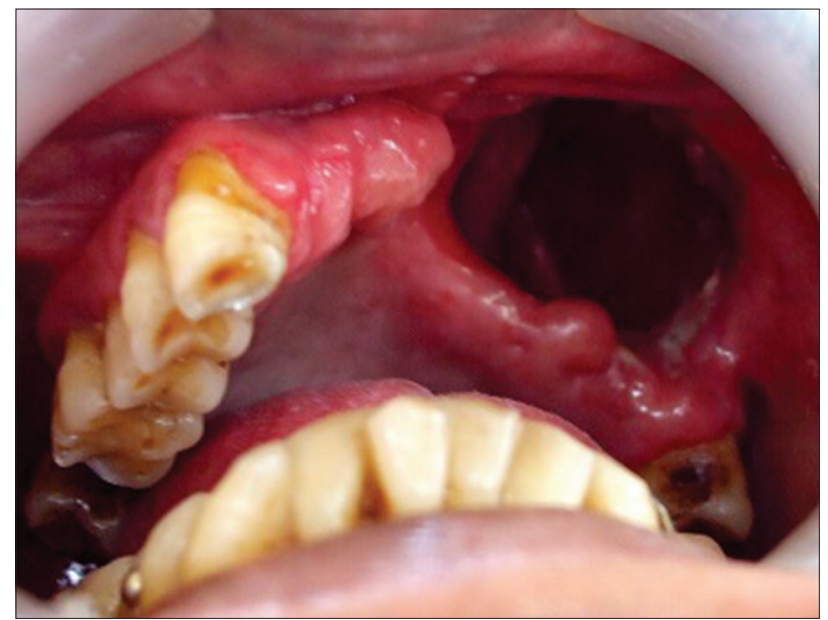

Figure 1: Intraoral view of the maxillectomy defect

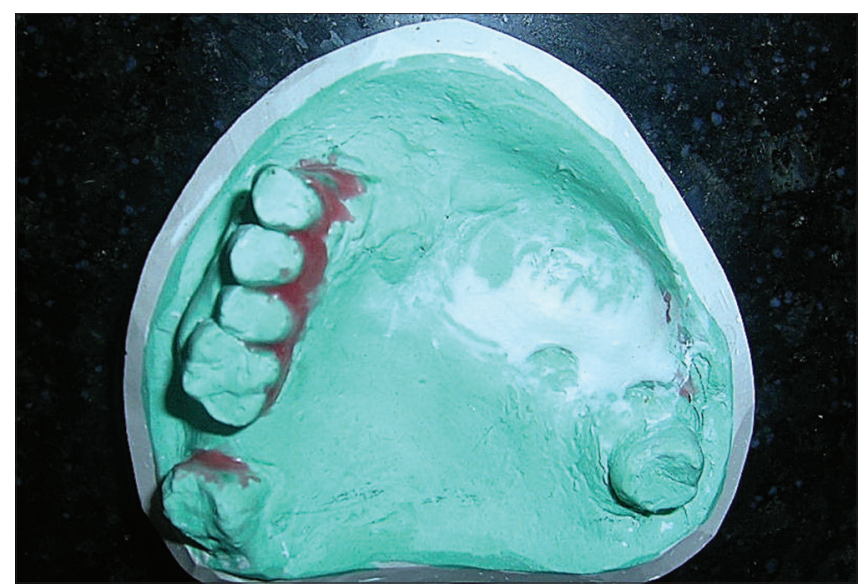

Figure 2: Blocking of undercuts on master cast by plaster and wax

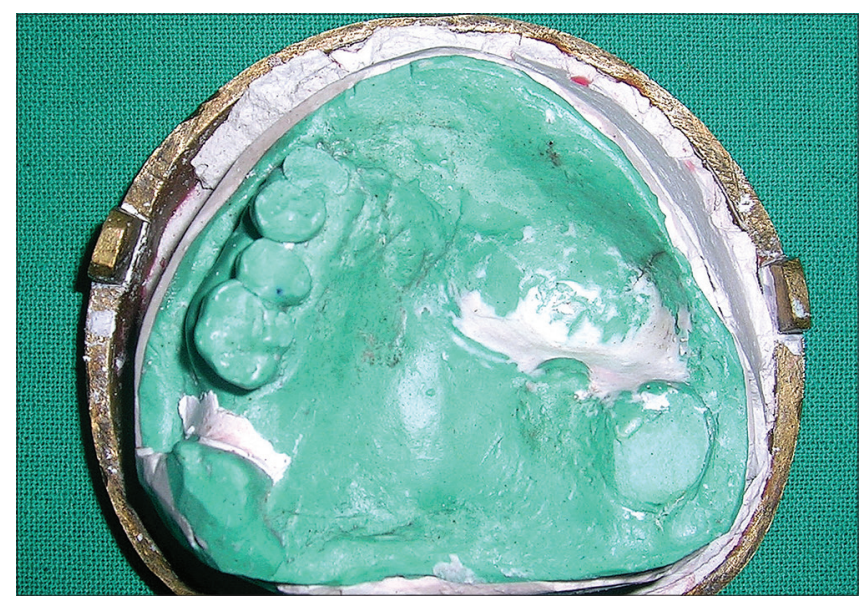

Figure 3: Master cast after elimination of wax

salt present in the bulb [Figure 7], resulting in a hollow space inside the bulb. The holes were sealed with a layer of self-curing acrylic, and final finishing and polishing of the prosthesis was done [Figure 8].

The plastic-based hollow obturator was inserted into the defect, and the patient was instructed on home care and the prosthesis maintenance. To sanitize the wound, the patient was instructed to gently remove any exudates with a wet cotton tip soaked with a $5 \%$ Betadine solution and to clean the intaglio surface of the prosthesis once 


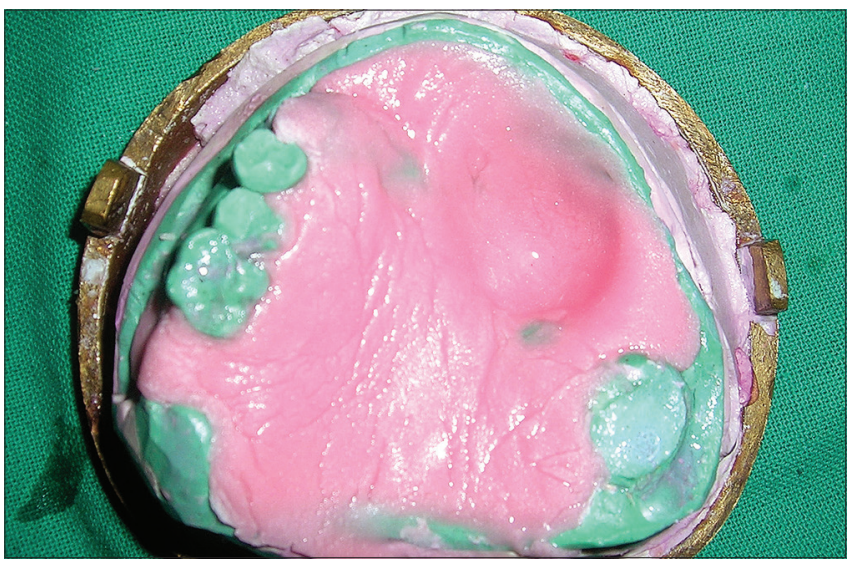

Figure 4: Plastic based polymethylmethacrylate heat cure placed and pressed into the defect

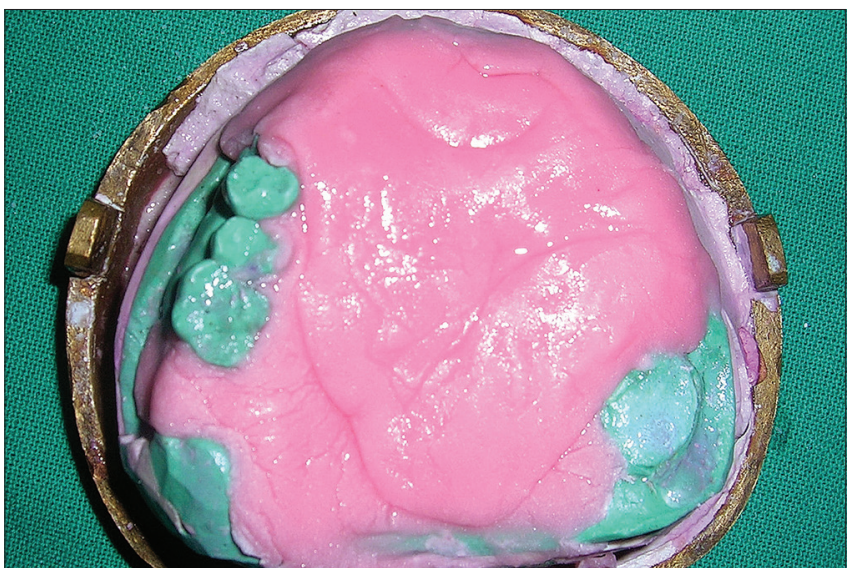

Figure 6: Second layer of plastic-based acrylic placed over the defect

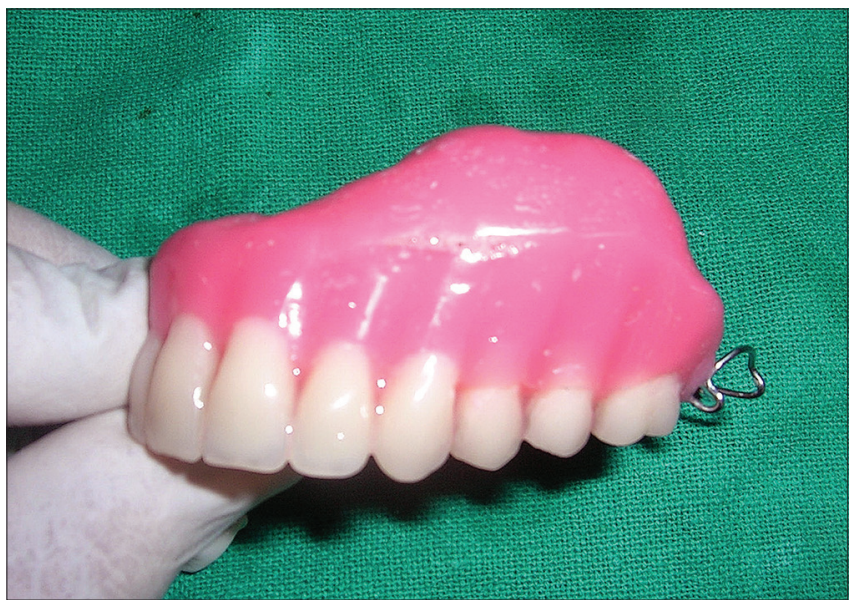

Figure 8: Finished and polished single unit hollow obturator closing the defect

a day. Post treatment photographs of the patient showed a marked improvement in aesthetics by replacement of missing teeth and restoration of the previously depressed nasomaxillary region [Figure $9 \mathrm{a}$ and b]. The patient was scheduled for his first adjustment 3 days following insertion. At the appointment, the surgical wound was examined to ensure health of the tissues and any part of prosthesis exerting pressure on the wound was smoothened. Hygiene and home care were emphasized, and the patient was advised to return in 3 months.

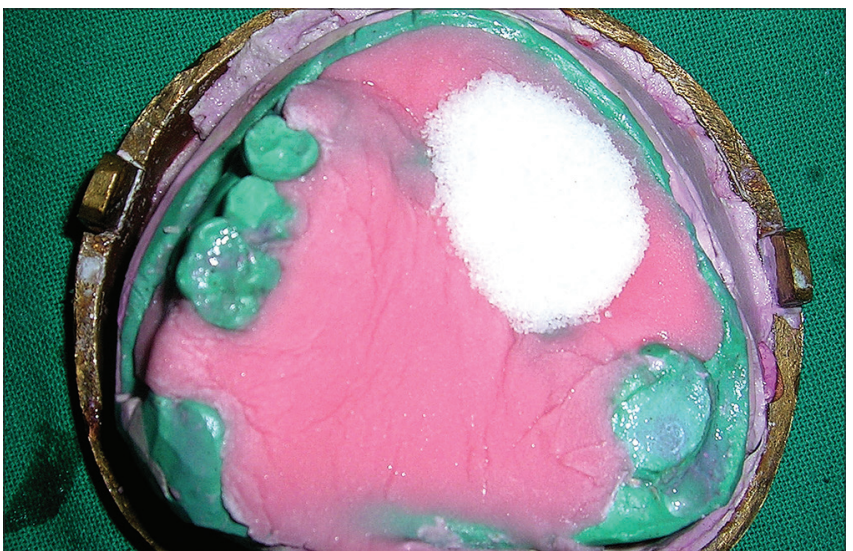

Figure 5: Salt filled into the defect

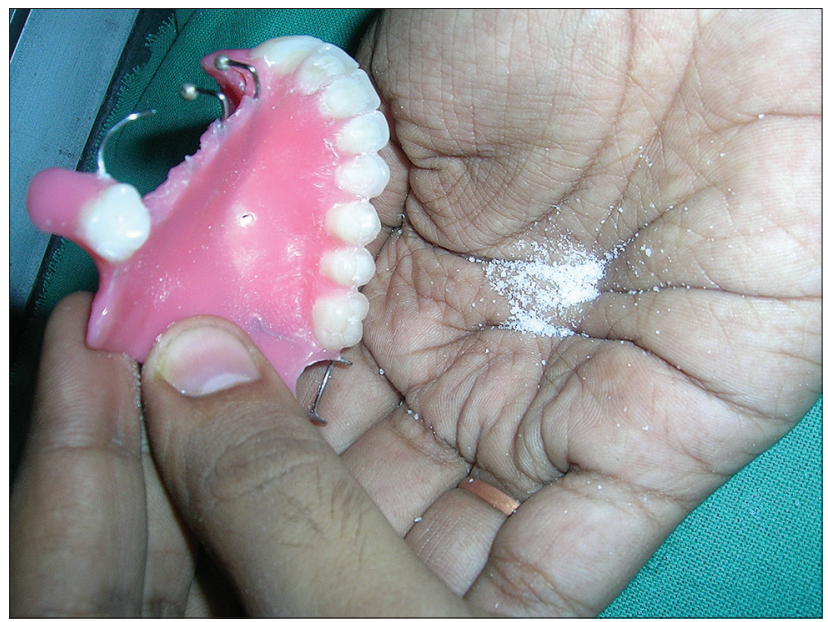

Figure 7: Elimination of salt through the holes

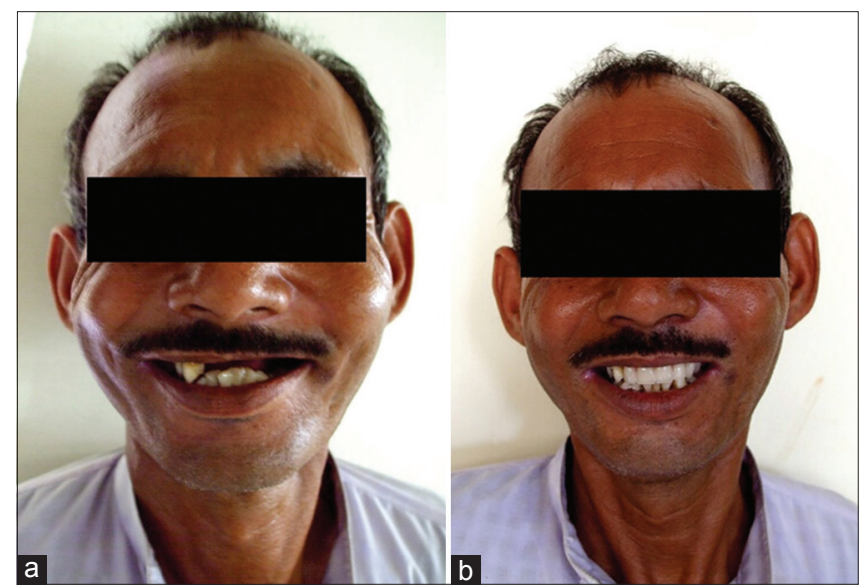

Figure 9: (a) Pretreatment photograph showing depressed left nasomaxillary region; (b) posttreatment photograph showing replaced missing teeth and marked improvement in aesthetics

\section{DISCUSSION}

Orofacial rehabilitation of patients with use of an obturator is an appropriate treatment modality for maxillofacial defects. ${ }^{[6]}$ Oromaxillary defects are associated with inflow and outflow of oral and nasal microflora, regurgitation of oral fluids, voice changes secondary to asynchrony in resonance, and difficulty in speech and swallowing. In addition, acquired maxillary defects have 
a marked effect on facial aesthetics. Hence, effective treatment modalities for these defects are mandatory. ${ }^{[7]}$ Small defects can be managed by surgery, but large defects require prosthodontic rehabilitation by obturators. A multidisciplinary team consisting of an oncologist, an oral and maxillofacial surgeon, a maxillofacial prosthodontist, a specialist nurse, a dietician and a speech therapist is ideal for care of head and neck cancer patients. A high level of cooperation between the prosthodontist and the surgeon prior to surgery is critical to achieving adequate rehabilitation for patients with maxillary defects. ${ }^{[8]}$

This clinical report describes the rehabilitation of a Class IV maxillary defect with a plastic based light weight hollow obturator. Class IV defects represent the classic maxillary defect in which the hard palate, alveolar ridge, and dentition are removed beyond the midline. ${ }^{[3]}$ Advantages of hollow bulb obturators include decreased weight of the obturator, decreased pressure on surrounding tissues, and ease of deglutition. In addition, the light weight of the obturator minimizes excessive atrophy and physiological changes in muscle balance. ${ }^{[9]}$ The hollow bulb adds resonance, thus improving the clarity of speech. ${ }^{[10]}$

Although prior techniques described in the literature fabricate obturators with the use of wax, sponge, polyurethane, foam and gas injection, ${ }^{[11-13]}$ the present technique uses a single-step flasking procedure, resulting in a comfortable, light-weight prosthesis with reduced fabrication time.

In conclusion, the goal of rehabilitation is creation of a prosthesis, which can restore aesthetics and function, while being easy to use, easy to clean to prevent recurrent infections, and which can be readily fabricated by simple time saving techniques. In order to achieve these goals, a single unit plastic based polymethylmethacrylate closed hollow obturator was fabricated by the lost salt method using single-step flasking. The prosthesis rehabilitated the patient aesthetically by replacing lost teeth and adding bulk to the depressed facial region, and functionally by providing better masticatory efficiency and phonetics. The present obturator is an additional alternative for plastic surgeons, oncologists and prosthodontists when planning treatment of such cases. In addition to being used following tissue healing, it can be used as an immediate surgical obturator by fabricating it on a presurgical model and trimming the affected area on the cast.

Educating and motivating the patient about the type of prosthesis and its limitations are the first steps in successful treatment. ${ }^{[14]}$ As this obturator is economical, time-saving and light in weight, the surgeon can recommend it to patients who require an economical alternative or who are not willing or able to undergo surgical reconstruction of their defect.

The light-weight plastic-based hollow bulb obturator fabricated in the present case rehabilitated the patient aesthetically and functionally, providing him an opportunity to live his life as close to normal as possible.

\section{REFERENCES}

I. Cummings CW. Cummings Otolaryngology: Head and Neck Surgery. 4th ed. St. Louis: Elsevier; 2004. p. 1604-8.

2. GPT-8. The academy of prosthodontics. J Prosthet Dent 2005;94:56.

3. Aramany MA. Basic principles of obturator design for partially edentulous patients. Part I: classification. J Prosthet Dent 1978;40:554-7.

4. Fornelli RA, Fedok FG, Wilson EP, Rodman SM. Squamous cell carcinoma of the anterior nasal cavity: a dual institution review. Otolaryngol Head Neck Surg 2000; 123:207-10.

5. Thawley SE, Batsakis JG, Lindberg RD, Panje WR, Donley S, editors. Comprehensive management of head and neck tumors. 2nd ed. St. Louis: Elsevier; 1998. p. 526-7.

6. Depprich R, Naujoks C, Lind D, Ommerborn M, Meyer U, Kübler NR, Handschel J. Evaluation of the quality of life of patients with maxillofacial defects after prosthodontic therapy with obturator prostheses. Int J Oral Maxillofac Surg 201 1;40:71-9.

7. Mohamed Usman JA, Ayappan A, Ganapathy D, Nasir NN. Oromaxillary prosthetic rehabilitation of a maxillectomy patient using a magnet retained two-piece hollow bulb definitive obturator; a clinical report. Case Rep Dent 2013;2013:190180.

8. Tirelli G, Rizzo R, Biasotto M, Di Lenarda R, Argenti B, Gatto A, Bullo F. Obturator prostheses following palatal resection: clinical cases. Acta Otorhinolaryngol Ital 2010;30:33-9.

9. Curtis TA, Beumer J. Restoration of acquired hard palate defects: etiology, disability, and rehabilitation. In: Beumer J, Curtis TA, Firtell DN, editors. Maxillofacial Rehabilitation. Prosthodontic and Surgical Considerations. St. Louis: C.V. Mosby Co.; 1979. p. 183-243.

10. Rilo B, Dasilva JL, Ferros I, Mora MJ, Santana U. A hollow-bulb interim obturator for maxillary resection. A case report. J Oral Rehabil 2005;32:234-6.

II. Patil PG, Patil SP. Fabrication of a hollow obturator as a single unit for management of bilateral subtotal maxillectomy. J Prosthodont 2012;2I:194-9.

12. Iramaneerat W, Seki F, Watanabe A, Mukohyama H, Iwasaki Y, Akiyoshi K, Taniguchi $\mathrm{H}$. Innovative gas injection technique for closed-hollow obturator. Int J Prosthodont 2004; I7:345-9.

13. Sridevi JR, Kalavathy N, Jayanthi N, Manjula N. Techniques for fabricating hollow obturator: two case reports. SRM J Res Dent Sci 2014;5:143-6.

14. Popli S, Parkash H, Bhargava A, Gupta S, Bablani D, Kar AK. A two-piece sectional interim obturator. A clinical report. J Prosthodont 20I2;2I:487-90.

How to cite this article: Bhatia V, Bhatia G. Aesthetic rehabilitation of a patient with an anterior maxillectomy defect, using an innovative single-step, single unit, plastic-based hollow obturator. Plast Aesthet Res 2015;2:140-3.

Source of Support: Nil, Conflict of Interest: None declared.

Received: 15-11-2014; Accepted: 15-03-2015 